\title{
LA VELOCIDAD EN ATLETAS JUVENILES DE DEPORTES DE CONJUNTO
}

Agudelo, C'1 Sanabria, Y², Flórez, J3

1. Carlos Alberto Agudelo Velásquez Mag. Profesor Ocasional de tiempo completo de la Universidad de Antioquia. Coordinador del pregrado Profesional en Entrenamiento Deportivo 2. Yofre Danilo Sanabria Arguello Mag. Profesor de tiempo completo de la Universidad Pedagógica y Tecnológica de Tunja. Coordinador de la Maestría Pedagogía de la Cultura Física. 3. Jairo Alberto Flórez Villamizar PhD. Profesor de tiempo completo de la Universidad Pedagógica y Tecnológica de Tunja. Escuela de Educación Física Recreación y deportes. Integrantes del grupo de investigación Tendencias Pedagógicas de la UPTC, grupo responsable de la investigación que origina el presente artículo.

\section{RESUMEN}

El presente artículo es fruto de un grupo de tres investigaciones basadas en realizar la preparación física aplicando un ATR de velocidad a deportes de conjunto: baloncesto, fútbol y voleibol, en deportistas de categorías juveniles, este trabajo da continuidad al realizado con jugadores de fútbol- sala de Tunja (Sanabria, 2013) y va en el sentido de construir la línea de investigación, frente a un interrogante genérico: ¿Es posible conseguir con ATR mejoras en velocidad en qué modalidades deportivas, con qué grupos etarios?, el presente trabajo trata de aclarar con evidencia estadística algunas de las aristas de este complejo interrogante.

Palabras clave: deportes de conjunto, desarrollo de la velocidad, atletas jóvenes, bloques concentrados.

\begin{abstract}
This article is the result of researchs performed based on physical preparation by applying a speed ATR team sports: basketball, football and volleyball at youth level athletes, this continues the work started with side football players of Tunja (Sanabria, 2013) and is in the sense of building research line, compared to a generic question: is it possible to achieve with ATR speed improvements in other embodiments, other groups and in other ages, this paper seeks to clarify with evidence statistical some of the edges of this complex question.
\end{abstract}

Keywords: team Sports, developing speed, young athletes, concentrated blocks. 


\section{INTRODUCCIÓN}

El presente artículo da cuenta de un grupo de investigaciones en deportes de conjunto que tienen como fin analizar la incidencia de la aplicación de ATR de ocho (8) semanas en deportistas juveniles para mejorar la velocidad 0 si se prefiere su velocidadagilidad, tal estudio se realiza con una población de 60 deportistas distribuidos así: 24 de baloncesto, 16 de fútbol y 20 de voleibol, los jugadores de baloncesto y voleibol fueron seleccionados de tal forma que cumplan 15 años en 2014 y los de fútbol 17, es decir los deportistas de cada modalidad tienen una edad biológica muy semejante ya que son nacidos en el mismo año y entre los grupos que se tomaron siempre pares se distribuyó aleatoriamente quienes realizaban la intervención y quienes se constituían en el grupo control, siempre en mitades iguales; las tareas propuestas como intervención reemplazaba el entrenamiento del grupo experimental, en tanto el grupo control continuo su preparación con los restantes integrantes del equipo, sólo los del grupo experimental desarrollaban durante las 8 semanas las tareas determinadas por el software, según su test inicial en la prueba de Illionois, reemplazando así tres (3) entrenamientos semanales.

Se realizó el test antes y después de la intervención, procurando que se hiciera en las mismas condiciones y se analizaron los resultados con el programa SPSS en su versión 12, encontrando que para los tres deportes y en las dos edades analizadas el programa aplicado tres veces a la semana por 8 semanas es efectivo ya que produce cambios estadísticamente significativos para mejorar la velocidad de desplazamiento en deportistas jóvenes de modalidades de conjunto.

La velocidad se puede considerar una de la base del rendimiento en muchas modalidades deportivas, en los deportes de conjunto, es muy evidente, ya que las acciones rápidas dan lugar al desarrollo de elementos defensivos y ofensivos y una mejor respuesta en el tiempo es un factor determinante para el rendimiento deportivo. El complejo particular en torno a la velocidad de cada modalidad es determinante (Weineck, 2005), en este pensamiento se puede resumir la gran incidencia que tiene la velocidad para el logro del rendimiento en la mayoría de deportes. Se encuentra también la afirmación de que la velocidad está determinada por las otras capacidades excepto cuando son acciones muy rápidas, además de que condicionan en los deportes de cooperación- oposición la toma decisiones y la técnica. (García, 1996).

La velocidad está en relación con la ejecución técnica, los factores motivacionales y obviamente con toda la estructura anatómico - cerebral se trata por tanto de una capacidad que puede ser clasificada tanto condicional como coordinativa (Grosser, 1991), este puede ser uno de los argumentos importantes para pensar que es posible aplicar métodos específicos de tipo contemporáneo 0 tradicional para el desarrollo de la velocidad en edades juveniles, ya que la latencia de lo coordinativo es precisamente en estas edades. La velocidad tiene una alta componente genética al depender en un gran porcentaje del tipo de fibra, sin embargo el mejoramiento de las denominadas cualidades de velocidad "puras" o "elementales" mejora con especial facilidad en la edad escolar (Weineck, 2005).

Dada las circunstancias especiales de la alta latencia del desarrollo de velocidad en las edades juveniles y afirmaciones como: "los principios de configuración en bloque y cambio brusco del volumen de la carga son validos para satisfacer las necesidades de especialización del entrenamiento en el deporte de élite y tal vez incluso en el de los jóvenes" (Martín, 2001) permiten un sustento para poder resolver el tal vez de 
Martin y experimentar con cargas en bloques de velocidad en edades juveniles. Es bien conocido que los sistemas de entrenamiento deben adaptarse a cada población y que desde la perspectiva de modelamiento se afirma que "no se trata de copiar modelos, lo que es necesario es adaptarlos a nuestras condiciones" (Agudelo, 2012), por ello trabajar con cargas tipo bloque en juveniles debe ser un proceso que permita tener en cuenta unas condiciones individuales de cada sujeto y otras grupales de cada equipo.

Los estudios referidos se hacen con base en adaptaciones creadas para poblaciones específicas, teniendo en cuenta sus condiciones propias de entrenamiento como la frecuencia y el volumen utilizado, lo que asegura que al tener poblaciones con características muy semejantes de entrenamiento, es posible medir objetivamente el efecto de un programa con cargas concentradas de velocidad. Desde hace mucho tiempo se afirma de la importancia que tiene adaptar a las circunstancias particulares de cada estado de desarrollo, insinuando que para ello es necesario investigar para organizar adecuadamente los procesos de entrenamiento (Verjoshanski, 1985).

El entrenamiento de la velocidad debe obedecer a una concentración limitada de objetivos, "cuando se trabaja en bloques las capacidades anaeróbicas es normal una gran acumulación de fatiga y por ende es indispensable reconocer la calidad de la respuesta de los deportistas" (Issurin,2012), por ello estos bloques de velocidad aplicados en esta investigación relegan otros objetivos de la preparación y controlan siempre durante su ejecución un adecuado porcentaje de la velocidad máxima a través de las cargas programadas por el software dándole aplicabilidad a la propuesta de Issurin, de adecuadas recuperaciones para este caso concreto de bloques de velocidad en edades juveniles.
Realizar el control de la velocidad resulta especialmente difícil, ya que los test son bastante inespecíficos, Meylan fue el primero en desarrollar test alrededor de los principios del siglo pasado, sobre las condiciones físicas: resistencia, rapidez y fuerza a través de evaluar las habilidades motrices elementales como: correr, trepar y saltar (Braun, 1980) y ya se aceptaba la dificultad de evaluar objetivamente una capacidad como la velocidad, en textos específicos de pruebas motrices se proponen evaluaciones diferenciales para: el tiempo de reacción, la capacidad de aceleración, el tiempo de movimiento, mantenimiento de la velocidad máxima, resistencia a la velocidad con pruebas entre 150 y 400 metros (García, 1996), lo que habla de la complejidad de evaluar esta capacidad, igualmente en Colombia en 2007 se proponen cerca de 10 pruebas para evaluar en niños y adolescentes esta capacidad ( Ramos, 2007), lo que refuerza el asunto de la dificultad de encontrar un test específico que pueda medir objetivamente las mejoras en velocidad como consecuencia de una intervención 0 planificación.

Para esta investigación se va a utilizar un test que es considerado de agilidad, ya que la agilidad es tomada como sinónimo de lo coordinativo (Weicneck,2005) y para medir las mejoras aplicables a la lógica de juego, desde la velocidad se puede pensar en la agilidad como esa combinación a veces un poco difícil de medir entre la velocidad y las respuestas rápidas que obedecen a la lógica interna de cada juego de conjunto.

El test de Illionois es por tanto el que se selecciona como instrumento de medición para verificar la evolución del entrenamiento de velocidad en los atletas de los grupos investigados. Este test tiene datos normativos que se encuentran disponibles en internet en forma de tablas como la siguiente: 


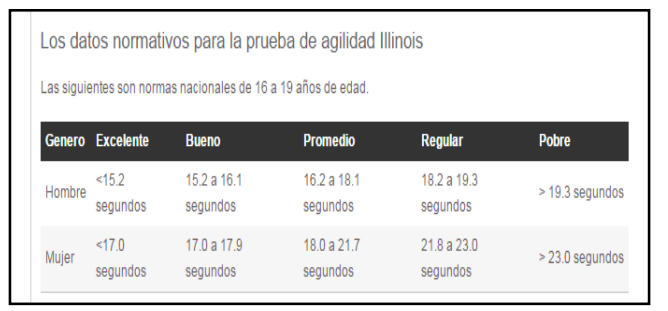

Tabla 1 Valores de referencia para el Test de illionois.

Recuperada de:

www.altorendimiento.com/tests-y calculadoras/calculadoras-deagilidad-paradeportistas/1241-prueba-de-agilidad-illinois.

Si bien el sitio en internet es español y la tabla a la que denominan norma nacional se supone es pensada para España, es un referente que permite tener un parámetro de comparación. Ya tiene un record del mundo que está en poder de Nick Wild (australiano), como figura en wikipedia con un tiempo de 10.28 impuesto en noviembre de 2011.

El protocolo, instrumentos y forma de ejecución de la prueba es bastante reconocida, este test por tanto pretende ser una valoración de las ganancias de velocidad que se pueda aplicar al juego, los tiempos de ejecución de las cargas y las distancias durante las ocho (8) semanas de intervención se consiguen desde los tiempos individuales de cada atleta y son un aporte del software creado en 2011 para entrenar salonistas. (Sanabria, 2013).

La forma general de ejecución del test se resume de la siguiente manera gráfica:

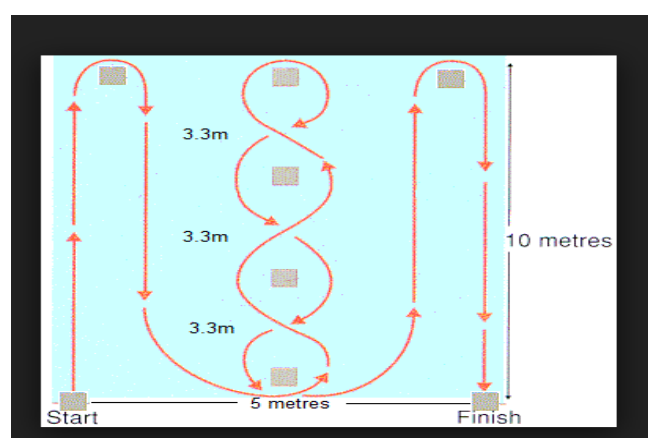

Gráfica1. Estructura general del test de illionois.
Recuperada dando test de Illinois graficas en google en:

https://www.google.com.co/search?q=test+de+illinois\&biw= 1366\&bih=643\&tbm=isch\&imgil=Vrey8mCEuuY_JM\%253A \%253BuvbnTfO8WNKFM\%253Bhttp\%25253A\%25252F\%2 5252Fwww.rehab.research.va.gov\%25252Fjour\%25252F2 013\%25252F507\%25252Fpage951.html\&source=iu\&pf $=m$ \&fir=Vrey8mCEuuY_JM\%253A\%252CuvbnTfO8WNKFM\% 252C_\&usg =_1p7z3CTpfu04Kyv0_RAjllciFSo\%3D\&ved= OCCYQyjc\&ei=GqiTVLLNKNPyggTkhYRg\#facrc=_\&imgdii= \&\&imgrc=Z5Of4m_Yu48tJM\%253A\%3BjXeyFWvosJPIGM \%3Bhttp\%253A\%252F\%252Fwww.brianmac.co.uk\%252Fp ictures $\% 252$ Ftests $\% 252$ Fillinois.gif\%3Bhttp $\% 253 \mathrm{~A} \% 252 \mathrm{~F}$ \%252Fwww.brianmac.co.uk\%252Fillinois.htm\%3B366\%3B 341

\section{METODOLOGÍA:}

Se utilizaron trabajos realizado por estudiantes de pregrado en la Licenciatura en Educación Física de la UPTC, que tuvieron asesoría directa de alguno de los integrantes del grupo de investigación Tendencias Pedagógicas los tres casos se documentan metodológicamente así:

Para los tres grupos se utiliza una intervención de ocho (8) semanas con un protocolo semejante de carga concentrada de ATR a través del software, trabajado previamente en 2011 por Sanabria, para jugadores de fútbol-sala, donde igual que en aquel estudio se evalúa el efecto de la intervención con el test de Agilidad de Illinois para describir las modificaciones estadísticas que tienen los grupos.

Cada grupo tiene su propio grupo control totalmente equiparable con el que se utilizó para la intervención, ya que surgen ambos, tanto el grupo experimental como el grupo control del mismo equipo.

La distribución de los jugadores se realizó de la siguiente manera, el equipo se separó aleatoriamente en dos mitades, previamente se elimino de tal sorteo los jugadores con: una edad diferente, baja asistencia, problemas de salud o desacuerdo con ser objetos de investigación. 
La hipótesis consiste en suponer que los cambios obtenidos en el pre-test y pos-test no son significativos y por ende la hipótesis alterna es considerar que los cambios en el grupo experimental si son significativos, previa demostración de que los grupos son comparables y de que además los cambios en el grupo NO intervenido son estadísticamente semejantes.

Igualmente se tuvieron todas las consideraciones éticas pertinentes así como el consentimiento y asentimiento informado por cada uno de los sujetos de investigación y sus respectivos representantes legales.

El tratamiento estadístico se va a realizar a través del programa SPSS, Versión 15,0 para Windows, en todos los casos se realizará una prueba de normalidad, en caso de que los datos obedezcan a una distribución normal se trabajará con pruebas $\mathrm{T}$ para las variables independientes relacionadas entre sí, de lo contrario se utilizarían pruebas paramétricas.

Con todas las condiciones anteriores los siguientes fueron los grupos intervenidos y su resultado:

Baloncesto: Jugadores de la categoría prejuvenil del Liceo Nacional José Joaquín Casas de la ciudad de Chiquinquirá 24 deportistas, 12 en el grupo control y 12 en el grupo experimental, todos de la misma edad 15 años cumplidos o por cumplir en 2014, es decir nacidos en 1999.

\section{GRUPO EXPERIMENTAL}

\begin{tabular}{|c|c|c|c|}
\hline & & $\begin{array}{l}\text { PRE- } \\
\text { TEST }\end{array}$ & $\begin{array}{l}\text { POS- } \\
\text { TEST }\end{array}$ \\
\hline DEPORTISTA & EDAD & TIEMPO & TIEMPO \\
\hline 1 & 15 & 17.73 & 14.09 \\
\hline 2 & 15 & 17.97 & 15,47 \\
\hline 3 & 15 & 17.61 & 15,65 \\
\hline 4 & 15 & 18,54 & 16,59 \\
\hline 5 & 15 & 17,85 & 15,97 \\
\hline 6 & 15 & 18,23 & 16,19 \\
\hline 7 & 15 & 18,47 & 17,28 \\
\hline $\mathbf{8}$ & 15 & 17.43 & 15,90 \\
\hline $\mathbf{9}$ & 15 & 17.95 & 16.21 \\
\hline 10 & 15 & 18,45 & 15,67 \\
\hline 11 & 15 & 17.66 & 15,43 \\
\hline 12 & 15 & 18,32 & 16,46 \\
\hline
\end{tabular}

Tabla 2 Valores pre-test y post test grupo experimental de Baloncesto.

\section{GRUPO CONTROL}

\begin{tabular}{|c|c|r|r|}
\hline & \multicolumn{2}{|c|}{$\begin{array}{c}\text { PRE- } \\
\text { TEST }\end{array}$} & $\begin{array}{r}\text { POS- } \\
\text { TEST }\end{array}$ \\
\hline DEPORTISTA & EDAD & TIEMPO & TIEMPO \\
\hline $\mathbf{1}$ & 15 & 18,83 & 18,98 \\
\hline $\mathbf{2}$ & 15 & 18,38 & 18,44 \\
\hline $\mathbf{3}$ & 15 & 17,69 & 17,73 \\
\hline $\mathbf{5}$ & 15 & 18,01 & 18,95 \\
\hline $\mathbf{6}$ & 15 & 17,94 & 17,03 \\
\hline $\mathbf{7}$ & 15 & 18,85 & 18,24 \\
\hline $\mathbf{8}$ & 15 & 18,43 & 18,60 \\
\hline $\mathbf{9}$ & 15 & 18,35 & 18,61 \\
\hline 10 & 15 & 17,64 & 17,10 \\
\hline 11 & 15 & 17,69 & 17,44 \\
\hline 12 & 15 & 17,23 & 17,42 \\
\hline & 15 & 17,81 & 18,75 \\
\hline
\end{tabular}

Tabla 3 Valores pre-test y post test grupo Control de Baloncesto.

\begin{tabular}{|c|c|c|c|c|c|}
\hline \multicolumn{3}{|c|}{ Grupo Experimental } & \multicolumn{3}{c|}{ Grupo Control } \\
\hline $\begin{array}{c}\text { Pre- } \\
\text { test }\end{array}$ & $\begin{array}{c}\text { post- } \\
\text { test }\end{array}$ & $\begin{array}{c}\text { Diferenci } \\
\text { a }\end{array}$ & $\begin{array}{c}\text { Pre- } \\
\text { test }\end{array}$ & $\begin{array}{c}\text { Post } \\
\text {-test }\end{array}$ & $\begin{array}{c}\text { Diferenci } \\
\text { a }\end{array}$ \\
\hline 17,7 & 14,0 & 3,64 & 18,9 & 18,8 & 0,15 \\
\hline 17,9 & 15,4 & 2,50 & 18,4 & 18,3 & 0,06 \\
\hline 18,5 & 15,6 & 2,89 & 17,7 & 17,6 & 0,04 \\
\hline 17,6 & 16,5 & 1,02 & 18,9 & 18,0 & 0,94 \\
\hline 18,2 & 15,9 & 2,26 & 17,0 & 17,9 & $-0,91$ \\
\hline 17,8 & 16,1 & 1,66 & 18,2 & 18,8 & $-0,61$ \\
\hline 18,4 & 17,2 & 1,19 & 18,6 & 18,4 & 0,17 \\
\hline 17,4 & 15,9 & 1,53 & 18,6 & 18,3 & 0,26 \\
\hline 17,9 & 16,2 & 1,74 & 17,1 & 17,6 & $-0,54$ \\
\hline 18,4 & 15,6 & 2,78 & 17,4 & 17,6 & $-0,25$ \\
\hline 17,6 & 15,4 & 2,23 & 17,4 & 17,2 & 0,19 \\
\hline 18,3 & 16,4 & 1,86 & 18,7 & 17,8 & 0,94 \\
\hline
\end{tabular}

Tabla 4: Diferencias entre el pre test y el post test de los deportistas del grupo experimental y del grupo control de Baloncesto.

Fútbol: Grupo formado por: 16 jugadores de Patriotas de 17 años divididos en dos subgrupos de 8 para los grupos experimental y control respectivamente, todos de 17 años para 2014 , es decir nacidos en 2007. 
GRUPO EXPERIMENTAL

\begin{tabular}{|c|c|c|c|}
\hline \multicolumn{2}{|c|}{} & PRE TEST & POST- \\
\hline DEPORTISTA & EDAD & TIEMPO & TIEMPO \\
\hline 1 & 17 & 14,4 & 13,8 \\
\hline 2 & 17 & 14,2 & 13.9 \\
\hline 3 & 17 & 14,1 & 13,7 \\
\hline 4 & 17 & 14,4 & 14,0 \\
\hline 5 & 17 & 14,6 & 14,1 \\
\hline 6 & 17 & 15,2 & 14,8 \\
\hline 7 & 17 & 14,7 & 14,4 \\
\hline 8 & 17 & 14,9 & 14,3 \\
\hline
\end{tabular}

Tabla 5: Resultados del grupo Experimental en Fútbol

\section{GRUPO CONTROL}

\begin{tabular}{|c|c|c|c|}
\hline & & PRE TEST & POS \\
\hline DEPORTISTA & EDAD & TIEMPO & TIEMPO \\
\hline 1 & 17 & 14,2 & 14,7 \\
\hline 2 & 17 & 15,2 & 15,7 \\
\hline 3 & 17 & 14,5 & 14,6 \\
\hline 4 & 17 & 14,8 & 14,10 \\
\hline 5 & 17 & 14,4 & 14,4 \\
\hline 6 & 17 & 14,5 & 14,4 \\
\hline 7 & 17 & 15,3 & 15,4 \\
\hline 8 & 17 & 14,9 & 15,1 \\
\hline
\end{tabular}

Tabla 6: Resultados del grupo Control en Fútbol

\begin{tabular}{|c|c|c|c|c|c|}
\hline \multicolumn{2}{|c|}{ Grupo experimental } & & Grupo & Control & \\
\hline Pre test & Post test & Diferencia & Pre test & Post test & Diferencia \\
\hline 14,4 & 13,80 & 0,60 & 14,20 & 14,70 & $-0,50$ \\
\hline 14,2 & 13,90 & 0,30 & 15,20 & 15,70 & $-0,50$ \\
\hline 14,1 & 13,70 & 0,40 & 14,50 & 14,60 & $-0,10$ \\
\hline 14,4 & 14,00 & 0,40 & 14,80 & 14,10 & 0,70 \\
\hline 14,6 & 14,10 & 0,50 & 14,40 & 14,40 & 0,00 \\
\hline 15,2 & 14,80 & 0,40 & 14,50 & 14,40 & 0,10 \\
\hline 14,7 & 14,40 & 0,30 & 15,30 & 15,40 & $-0,10$ \\
\hline 14,9 & 14,30 & 0,60 & 14,90 & 15,10 & $-0,20$ \\
\hline
\end{tabular}

Tabla 7: Diferencias entre el pre test y el post test de los deportistas del grupo experimental y del grupo control.
Voleibol: Grupo formado por 20 sujetos del club de Voleibol denominado Profesionales de la ciudad de Tunja, 10 en el grupo experimental y 10 en el grupo control, todos nacidos en 1999, es decir que cumplen 15 años en 2014.

\section{GRUPO EXPERIMENTAL}

\begin{tabular}{|c|c|c|c|}
\hline & & $\begin{array}{l}\text { PRE- } \\
\text { TEST }\end{array}$ & $\begin{array}{l}\text { POS- } \\
\text { TEST }\end{array}$ \\
\hline DEPORTISTA & EDAD & TIEMPO & TIEMPO \\
\hline 1 & 15 & 15,836 & 14,773 \\
\hline 2 & 15 & 15,346 & 14,531 \\
\hline 3 & 15 & 15,547 & 14,508 \\
\hline 4 & 15 & 15,363 & 14,761 \\
\hline 5 & 15 & 15,970 & 14,983 \\
\hline 6 & 15 & 15,600 & 14,509 \\
\hline 7 & 15 & 16,346 & 14,830 \\
\hline 8 & 15 & 15,464 & 14,780 \\
\hline 9 & 15 & 15,117 & 14,525 \\
\hline 10 & 15 & 15,072 & 14,917 \\
\hline 11 & 15 & 15,836 & 14,773 \\
\hline 12 & 15 & 15,346 & 14,531 \\
\hline
\end{tabular}

Tabla 8: Valores pre-test y post test grupo experimental de Voleibol.

GRUPO CONTROL

\begin{tabular}{|r|r|r|r|}
\hline & & \multicolumn{1}{c|}{$\begin{array}{r}\text { PRE- } \\
\text { TEST }\end{array}$} & \multicolumn{1}{c|}{$\begin{array}{c}\text { POS- } \\
\text { TEST }\end{array}$} \\
\hline DEPORTISTA & EDAD & TIEMPO & TIEMPO \\
\hline $\mathbf{1}$ & 15 & 15,189 & 15,126 \\
\hline $\mathbf{2}$ & 15 & 15,489 & 15,523 \\
\hline $\mathbf{3}$ & 15 & 16,206 & 14,756 \\
\hline $\mathbf{4}$ & 15 & 15,597 & 15,621 \\
\hline $\mathbf{5}$ & 15 & 15,682 & 15,572 \\
\hline $\mathbf{6}$ & 15 & 15,106 & 15,759 \\
\hline $\mathbf{7}$ & 15 & 15,889 & 15,846 \\
\hline $\mathbf{8}$ & 15 & 15,588 & 15,698 \\
\hline $\mathbf{9}$ & 15 & 15,844 & 15,423 \\
\hline $\mathbf{1 0}$ & 15 & 15,769 & 16,135 \\
\hline $\mathbf{n}$ & & & \\
\hline
\end{tabular}

Tabla 9: Valores pre-test y post test grupo Control de Voleibol 


\begin{tabular}{|r|r|r|r|r|r|}
\hline \multicolumn{2}{|c|}{ Grupo Experimental } & \multicolumn{3}{c|}{ Grupo Control } \\
\hline $\begin{array}{r}\text { Pre- } \\
\text { test }\end{array}$ & $\begin{array}{c}\text { post- } \\
\text { test }\end{array}$ & Diferencia & $\begin{array}{r}\text { Pre- } \\
\text { test }\end{array}$ & Post-test & Diferencia \\
\hline 15,836 & 14,773 & 1,063 & 15,189 & 15,126 & $-0,063$ \\
\hline 15,346 & 14,531 & 0,815 & 15,489 & 15,523 & 0,034 \\
\hline 15,547 & 14,508 & 1,039 & 16,206 & 14,756 & $-1,450$ \\
\hline 15,363 & 14,761 & 0,602 & 15,597 & 15,621 & 0,024 \\
\hline 15,970 & 14,983 & 0,987 & 15,682 & 15,572 & $-0,110$ \\
\hline 15,600 & 14,509 & 1,091 & 15,106 & 15,759 & 0,653 \\
\hline 16,346 & 14,830 & 1,516 & 15,889 & 15,846 & $-0,043$ \\
\hline 15,464 & 14,780 & 0,684 & 15,588 & 15,698 & 0,110 \\
\hline 15,117 & 14,525 & 0,592 & 15,844 & 15,423 & $-0,421$ \\
\hline 15,072 & 14,917 & 0,155 & 15,769 & 16,135 & 0,366 \\
\hline
\end{tabular}

Tabla10: Diferencias entre el pre test y el post test de los deportistas del grupo experimental y del grupo control de Voleibol

\section{ANALISIS DE LOS RESULTADOS}

Los resultados fueron analizados de la siguiente forma: se midió si los datos del pre-test son comparables, para asegurar que la investigación no se sesga desde el comienzo, para tal fin se hace una prueba de normalidad entre los datos del pre-test de ambos grupos, como se encontró para los tres (3) grupos que los datos son normales se relacionaron las variables a través de una Prueba T, para mirar si se dieron cambios importantes entre las muestras relacionadas.

Para los tres deportes se relacionaron entre sí las siguientes parejas de datos:

Los datos de los dos pre-test: grupo control y grupo experimental (preTestE/preTestC)

Los datos de los dos pos-test: grupo control y grupo experimental (posTestE/posTestC)

Datos del pre-test y el postest del grupo Experimental (preTestE/preTestE)

Datos del pre-test y el postest del grupo Control (preTestC/preTestC)

Grupo de Baloncesto
Prueba de Normalidad:

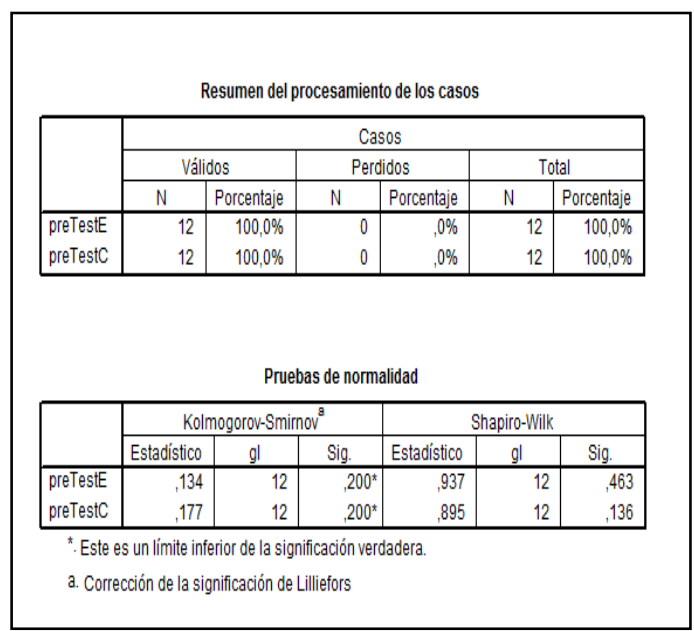

Tabla11: Datos normales al ser la significancia superior a 0.05 (0.436 y 0.136 respectivamente)

Pruebas T.

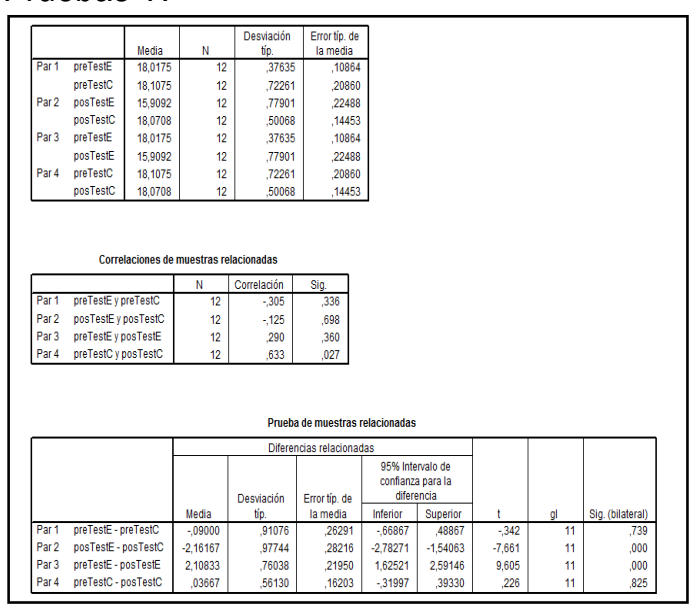

Tabla 12: Prueba T para muestras independientes en el pre-test de baloncesto.

Por tanto para este grupo de baloncesto se observa que:

Los valores iniciales son básicamente semejantes, pre-test de ambos grupos (0.739)

Los valores del pos-tetst en los dos grupos son diferentes $(0.000)$

Las modificaciones del pre-test y pos-test para el grupo experimental es estadísticamente significativas.(0.000) 
Las modificaciones del pre-test y pos-test para el grupo control no es significativas.(0.825)

Grupo de futbolistas de las inferiores de Patriotas

Prueba de Normalidad:

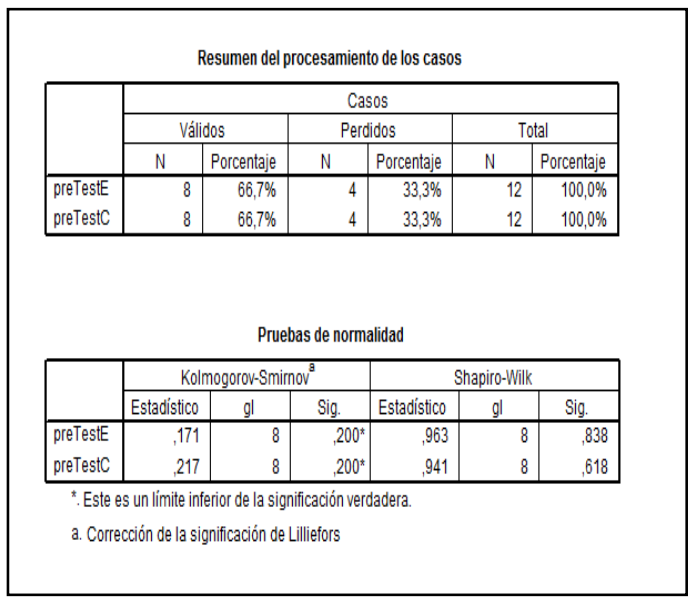

Tabla13: Datos normales al ser la significancia superior a 0.05 (0.838 y 0.618 respectivamente)

Pruebas T para las variables relacionadas:

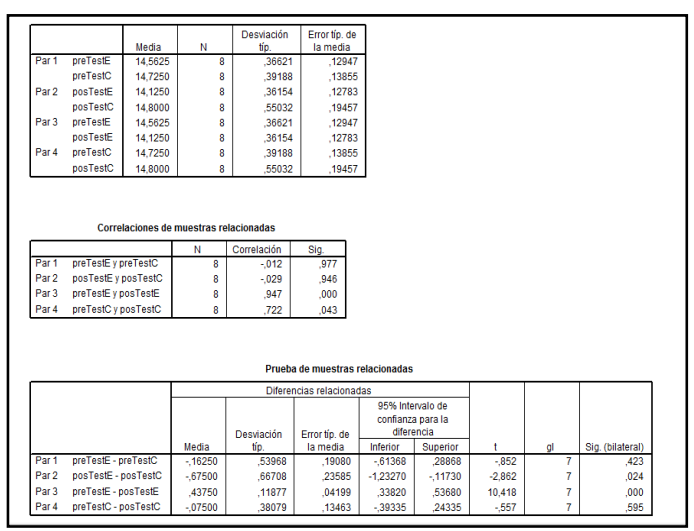

Tabla 14: Prueba T para muestras independientes en el pre-test de fútbol.

Por tanto para este grupo de fútbol se observa que:

Los valores iniciales son básicamente semejantes, pre-test de ambos grupos $(0.423)$.
Los valores del post-test en los dos grupos son diferentes $(0.024)$.

Las modificaciones del pre-test y pos-test para el grupo experimental es estadísticamente significativas.(0.000).

Las modificaciones del pre-test y pos-test para el grupo control no es significativas.(0.595).

Grupo de voleibol

Prueba de normalidad de los datos del pretest

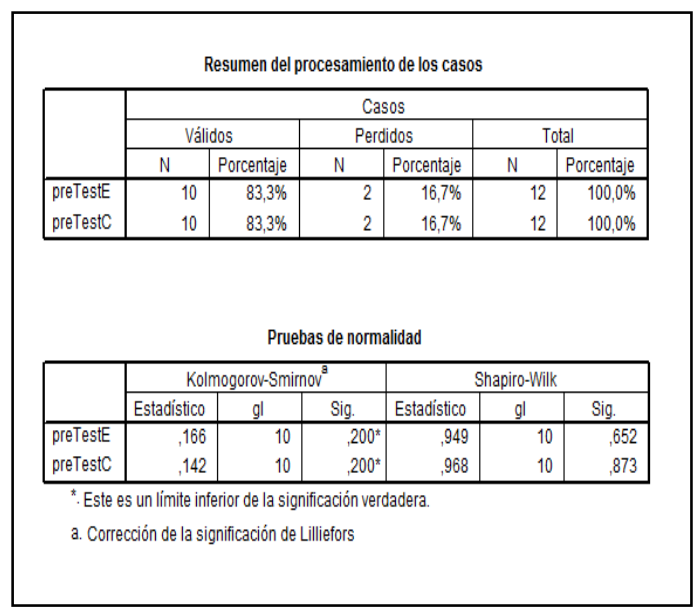

Tabla15: Datos normales al ser la significancia superior a 0.05 (0.652 y 0.873 respectivamente)

Resultados de la Prueba T para variables relacionadas:

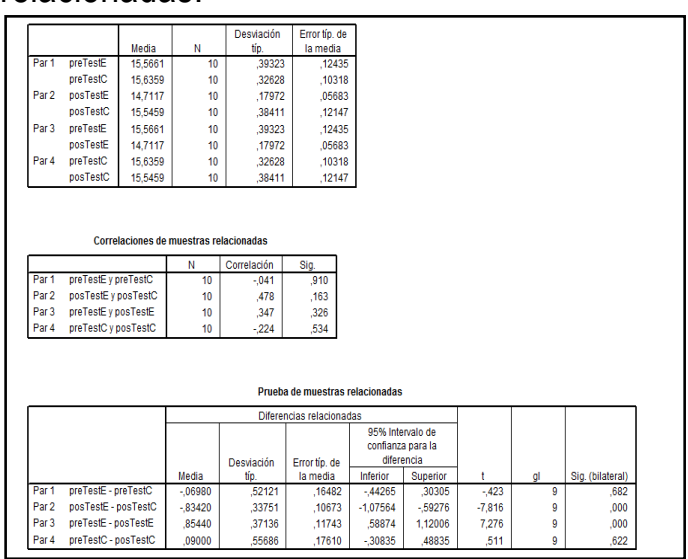

Tabla 16: Prueba T para muestras independientes en el pre-test de voleibol. 
Por tanto para este grupo de voleibol se observa que:

Los valores iniciales son básicamente semejantes, pre-test de ambos grupos (0.682).

Los valores del post-test en los dos grupos son diferentes $(0.000)$.

Las modificaciones del pre-test y pos-test para el grupo experimental es estadísticamente significativas.(0.000).

Las modificaciones del pre-test y pos-test para el grupo control no es significativas.(0.622).

\section{CONCLUSIONES Y RECOMENDACIONES}

Se puede considerar que es válido el uso de bloques concentrados de velocidad para desarrollar la agilidad en jugadores jóvenes de deportes de conjunto, de acuerdo con las recomendaciones de Verjonsanski de investigar para poder programar mejor los procesos de entrenamiento, se podría afirmar que este proceso dió respuesta a esta inquietud en el sentido que permite afirmar que es positivo el uso de bloques de velocidad para jóvenes atletas de deportes de conjunto.

Resulta también verificable la afirmación de la latencia del desarrollo de la velocidad que plantea Weicneck, para deportistas juveniles ya que las edades de 15 años en voleibolistas y baloncestistas, así como la de 17 para futbolistas permitieron resultados positivos al aplicar cargas concentradas de velocidad.

Las insinuaciones de adaptar los sistemas de planificación internacionales a lo local de Agudelo, tienen en este trabajo un ejemplo positivo ya que con tales presupuestos se pudo encontrar un sistema de desarrollo de la velocidad que mostró resultados para este grupo de investigaciones.
El tal vez de Martin en: Manual de Metodología del entrenamiento deportivo (2001) quedaría resuelto favorablemente desde los hallazgos de esta investigación, ya que en estos casos resulto positivo utilizar los bloques para trabajar con atletas jóvenes en la idea de desarrollarles su velocidad.

Se demuestra que el software para construir bloques de tareas de velocidad creado por Sanabria respeta las necesidades de recuperación planteadas para este tipo de aplicación de cargas de Issurin, dados los resultados a los que se llegan.

Un factor importante que se tuvo para el trabajo puede haber estado determinado por la rigurosidad en la elaboración de los grupos, que aseguro edades y niveles de desarrollo semejantes, intervenciones replicadas con un gran rigor y un permanente seguimiento a las intervenciones, lo que pudo haber evitado factores intervinientes desfavorables.

Se crea la necesidad de verificar si puede tener validez para otras edades u otro tipo de modalidades deportivas, lo que constituye una necesaria continuidad del presente tipo trabajos investigativos en el área de de la velocidad.

\section{REFERENTES BIBLIOGRÁFICOS}

Agudelo C. (2012) Entrenamiento Deportivo por Modelamiento. Editorial Kinesis. Armenia- Colombia.

Braun H. (1980) Convenio colombo alemán de educación física, deporte y recreación. Tomo 6: Teoría y Práctica de los test deportivos motores. Editorial $\mathrm{XYZ}$. Bogotá - Colombia.

García J; Navarro M; Ruiz J. (1996) Pruebas para la valoración de la capacidad motriz en el deporte. Gymnos editorial deportiva. Madrid- España.

Grosser M. (1991) Entrenamiento de la velocidad. 
Issurin V. (2012) Entrenamiento Deportivo periodización en Bloques. Editorial Paidotribo. Barcelona - España.

Martín D. (2001) Manual de metodología del entrenamiento deportivo. Editorial Paidotribo. Barcelona - España.

Ramos S; Melo L; Alzate D. (2007) Evaluación antropométrica y motriz condicional de niños y adolescentes. Editorial uiversidad de Caldas. Manizales Colombia.

Sanabria Y. (2013) Futbol sala. La velocidad de desplazamiento con velocidad ATR. Editorial Kinesis. Armenia- Colombia.

Verjoshanski. (1985) Entrenamiento Deportivo. Planificación y Programación. Cultura física y deporte. Moscú - Rusia.

Weineck J. (2005) Entrenamiento Total. Editorial Paidotribo. Barcelona - España. 